\title{
Space Frequency Codes from Spherical Codes
}

\author{
Oliver Henkel \\ Fraunhofer German-Sino Lab for Mobile Communications - MCI \\ Einsteinufer 37, 10587 Berlin, Germany \\ Email: henkel@hhi.fraunhofer.de
}

\begin{abstract}
A new design method for high rate, fully diverse ('spherical') space frequency codes for MIMO-OFDM systems is proposed, which works for arbitrary numbers of antennas and subcarriers. The construction exploits a differential geometric connection between spherical codes and space time codes. The former are well studied e.g. in the context of optimal sequence design in CDMA systems, while the latter serve as basic building blocks for space frequency codes. In addition a decoding algorithm with moderate complexity is presented. This is achieved by a lattice based construction of spherical codes, which permits lattice decoding algorithms and thus offers a substantial reduction of complexity.
\end{abstract}

\section{INTRODUCTION}

In MIMO (Multiple Input Multiple Output) systems space time coding schemes have been proven to be an appropriate tool to combat fading holes of Rayleigh flat fading channels and exploit the spatial diversity gains. For frequency selective SISO (Single Input Single Output) channels (due to multipath propagation) OFDM (Orthogonal Frequency Division Multiplexing) provides an efficient strategy to obtain a collection of mutually orthogonal flat fading channels on each subcarrier. The number $K$ of subcarriers in such a system is not a free design parameter but predetermined by the bandwidth (which determines the sampling rate) and the coherence length of the channel (which determines the maximal OFDM symbol length, which is the maximum number of subcarriers). MIMO-OFDM is the combination of MIMO techniques to frequency selective Rayleigh (multipath) channels. Space frequency coding aims to combine the advantages of space time coding with the multipath diversity from each channel tap.

A corresponding code design for fully diverse space frequency codes $\mathcal{C}$ has been proposed in [1]. In their work they separated the code construction into the design of an appropriate transition matrix $A$ incorporating the multipath diversity and the construction of a corresponding space time code $\tilde{\mathcal{C}}$. This method has the advantage of splitting up the design problem into two separated problems by keeping the multipath echoes of the transmit signal mutually orthogonal. For these reasons this work focuses on the design method [1], although other approaches have been investigated (e.g. [2]).

To obtain maximum rates by retaining full (spatial and frequency) diversity order the block length $T$ of the 'inner' space time code has to be as large as possible (i.e. $T=K / L$, whereas $L$ denotes the number of taps). This requirement rules out most of the space time code designs considered in the literature so far. Common research has been focused mainly on small dimensional coding spaces (i.e. $T=n_{t}$ ) whose elements consist of square $n_{t} \times n_{t}$ matrices, whereas $n_{t}$ denotes the number of transmit antennas. These designs correspond to the case $K / L=n_{t}$ only. To attack the more general (space time) code design problem an inspection of the maximum likelihood decision criterion reveals, that it is a good first approach to consider code design as a packing problem. The corresponding coding space turns out to be the complex Stiefel manifold of complex $T \times n_{t}$ matrices with unit norm mutually complex orthogonal column vectors.

On the other hand the design of optimal (Welch bound equality WBE) signature sequences in CDMA (Code Division Multiple Access) leads naturally to a packing problem on spheres [3], [4]. Explicit constructions of spherical packings can be found on N.J.A. Sloane's web page ${ }^{1}$.

The first achievement of this work is to employ differential geometric methods to establish a connection between spherical packings/codes and (general) space time packings/codes, such that the rich variety of already known spherical codes can be transformed to space time codes in various dimensions. This mapping preserves (geodesic) distances, and therefore the performance at the receiver can be controlled (up to a certain degree). The so constructed codes are called spherical space time codes and the resulting space frequency codes (obtained from the procedure described above) are named spherical space frequency codes from now on.

The second part of this work is concerned with decoding strategies. The need for high rate code designs demands advanced decoding algorithms. Even moderate numbers $K$ of subcarriers need huge codebooks of size $2^{K R}$ in order to retain a specified rate $R$, making brute force maximum likelihood decoding impossible for practical applications. Based on wrapped spherical codes [5] which are decodable in terms of lattice algorithms, the connection to space time codes will be exploited to do the same for space time and space frequency codes. This preferred decoding strategy prohibits high rate spherical code classes obtained elsewhere, such as Sloane's dense packings or packings obtained from frame theory (e.g. [6]).

On the other hand wrapped spherical coding offers a wide range of lattice based constructions which can be adapted to arbitrary numbers of antennas and subcarriers. Moreover for each lattice of the appropriate dimension a target minimal distance can be prescribed, leading to a corresponding wrapped spherical code which can be processed further to obtain space time and space frequency codes. In this way whole (fully diverse) families of codes can be constructed for each prescribed number of antennas and subcarriers, whereas the

${ }^{1}$ www. research. att. com/ n jas/packings/ 
rate can be adapted by choosing appropriate minimal distances.

The text is structured as follows. In the next two sections the channel model and the basic code design criteria will be derived. Section IV is devoted to the design of space frequency codes, which has been made popular in [1]. Section V sketches the differential geometric connection between spherical codes and space time codes and describes the corresponding mapping. In section VI the second part of this work starts. It contains the encoding and decoding procedure (based on [5] for the spherical part) for the proposed spherical space frequency codes, which are simulated in section VII. Finally in the last section the results are summarized for a quick reference.

\section{CHANNEL MODEL}

Let us adopt the following notations: $\hat{x}=\operatorname{FFT}(x), x^{\prime}=$ $\operatorname{IFFT}(x)=\operatorname{FFT}^{\dagger}(x),-$ denotes complex conjugation, ${ }^{t}$ the transpose, ${ }^{\dagger}$ the hermitian transpose, 1 denotes the identity matrix and the all-zero matrix is denoted as $\mathbf{0}$, whereas their dimensions will be clear from the context, if not indicated by some subscripts. Using the common (complex conjugated) $K$-th root of unity $\omega=e^{-\frac{2 \pi \imath}{K}}$ the ( $K$-point) (I)FFT matrix reads $(\mathrm{I}) \mathrm{FFT}=\left[(\mathrm{I}) \mathrm{FFT}_{k k^{\prime}}\right]_{k, k^{\prime}=0}^{K-1}$ with components $\mathrm{FFT}_{k k^{\prime}}=$ $\frac{1}{\sqrt{K}} \omega^{k k^{\prime}}$, resp. IFFT $_{k k^{\prime}}=\frac{1}{\sqrt{K}} \bar{\omega}^{k^{\prime} k}$. The frequently used operations of (frequency) modulation $\mu=\operatorname{diag}\left(\omega^{k}\right)_{k=0}^{K-1}$ and (time) translation $\tau=\left[e_{(k+1) \bmod K}\right]_{k=0}^{K-1}$ on $\mathbb{C}^{K}\left(e_{k}\right.$ denoting the $k$-th standard basis vector) transform into each other by $\mu=\mathrm{FFT} \circ{ }^{\circ} \circ \mathrm{IFFT}$, resp. $\tau=$ IFFT $\circ \mu \circ \mathrm{FFT}$.

The channel model assumed here is the frequency selective Rayleigh fading MIMO-OFDM channel with $K$ subcarriers, $L$ taps $H_{l} \in \mathbb{C}^{n_{t} \times n_{r}} \sim$ i.i.d. $\mathcal{C N}\left(0, \frac{1}{L}\right)$, and $n_{t}$, (resp. $n_{r}$ ) transmit (resp. receive) antennas. Before transmission a $K$ point IFFT is applied to each $K \times n_{t}$ MIMO-OFDM code symbol $C=\left(c_{0}^{t}, \ldots, c_{K-1}^{t}\right)^{t}$, which is then prepended by the cyclic prefix of length $L$. The receiver applies a $K$ point FFT and removes the cyclic prefix before (maximum likelihood) decoding. Then, the transmission equation reads in the frequency domain

$$
\tilde{c}_{k}=\sqrt{\rho} c_{k} \hat{H}_{k}+n_{k}, k=0, \ldots, K-1
$$

whereas

$$
\hat{H}_{k}=\sum_{l=0}^{L-1} H_{l} e^{-\frac{2 \pi \imath}{K} k l}
$$

and $n_{k} \in \mathbb{C}^{1 \times n_{r}}$ (noise), with both $\hat{H}_{k}$ and $n_{k}$ having i.i.d. $\mathcal{C N}(0,1)$ distributed components for all $k=0, \ldots, K-1$. It is assumed that the transmitter knows only the channel statistic while the receiver perfectly knows the channel coefficients. The rows $c_{k}$ of $C$ are assumed to have unit norm, such that $\rho$ is the SNR (signal to noise ratio) per subcarrier at each receive antenna.

\section{SPACE FREQUENCY CODE DESIGN CRITERIA}

This section describes the code design criteria developed in [1] with emphasis on the underlying geometrical framework.
Let us express the transmitted symbols $C$ as $C:=\sqrt{\rho \frac{K}{n_{t}}} \Phi$, assuming $\Phi$ to be an element of the complex Stiefel manifold

$$
V_{n_{t}, K}^{\mathbb{C}}=\left\{\Phi \in \mathbb{C}^{K \times n_{t}} \mid \Phi^{\dagger} \Phi=\mathbf{1}\right\}
$$

which is the basic coding space from now on, compare [7],[1]. A space frequency code is given by a discrete set $\mathcal{C} \subset V_{n_{t}, K}^{\mathbb{C}}$ and provided the received signal $\tilde{C}=\sqrt{\rho \frac{K}{n_{t}}} \Psi H+N$ the maximum likelihood decision reads (see [8])

$$
\Phi_{\mathrm{ML}}=\arg \min _{\forall \Phi \in \mathcal{C}}\left\|\tilde{C}-\sqrt{\rho \frac{K}{n_{t}}} \Phi H\right\|_{\mathrm{F}}
$$

In the sequel let us adopt the notation that in order to emphasize the space Frequency (code) or space Time (code) interpretation of a given quantity subscripts ${ }_{F}$, respectively $\cdot_{T}$ will be added. Further, for the difference symbol $\Delta=$ $\Phi-\Psi$ of two codesymbols we define the 'multipath extension' $\Delta_{\text {mp }}=\left(\Delta, \mu \Delta, \ldots, \mu^{L-1} \Delta\right)$. Then the Chernov bound for the probability of mistaking $\Phi$ for $\Psi$ is given by (compare [1])

$$
\operatorname{ch}_{F}=\left(\prod_{i=1}^{n_{t} L}\left[1+\varrho_{F} \sigma_{i}^{2}\left(\Delta_{\mathrm{mp}}\right)\right]\right)^{-n_{r}}
$$

whereas $\varrho_{F}:=\frac{1}{4} \rho \frac{K}{n_{t} L}$ and $\sigma(M)=\left(\sigma_{i}(M)\right)$ generically denotes the vector of singular values of the matrix $M$ in decreasing order. Expanding the product leads to the diversity, resp. diversity sum $\mathrm{d}_{F}$ and product $\mathrm{p}_{F}$

$$
{\mathcal{D} i v_{F}}:=\prod_{i=1}^{n_{t} L}\left[1+\varrho_{F} \sigma_{i}^{2}\left(\Delta_{\mathrm{mp}}\right)\right]=\sum_{i=0}^{n_{t} L} s_{i} \varrho_{F}^{i}
$$

whereas $\left(\operatorname{sym}_{i}\right.$ denotes the $i$-th elementary symmetric polynomial) $s_{i}:=\operatorname{sym}_{i}\left(\sigma_{1}^{2}\left(\Delta_{\mathrm{mp}}\right), \ldots, \sigma_{n_{t} L}^{2}\left(\Delta_{\mathrm{mp}}\right)\right)$ and

$$
\begin{gathered}
\mathrm{d}_{F}^{2}:=s_{1}=\left\|\Delta_{\mathrm{mp}}\right\|_{\mathrm{F}}^{2}=L\|\Delta\|_{\mathrm{F}}^{2}=L \mathrm{~d}_{T}^{2} \\
\mathrm{p}_{F}^{2}:=s_{n_{t} L}=\operatorname{det}\left(\Delta_{\mathrm{mp}}^{\dagger} \Delta_{\mathrm{mp}}\right) \leq\left(\mathrm{p}_{T}^{2}\right)^{L}
\end{gathered}
$$

with $\mathrm{d}_{T}:=\|\Delta\|_{\mathrm{F}}, \mathrm{p}_{T}:=\sqrt{\operatorname{det} \Delta^{\dagger} \Delta}$ denoting the corresponding diversity sum and diversity product for space-time codes. Note, that due to $\mathrm{d}_{F}^{2}=L \mathrm{~d}_{T}^{2}$ the maximum likelihood detection for space frequency codes is essentially the same than for space time codes. Further, equality in (Fischer's inequality) (8) holds, if $\Delta^{\dagger} \mu^{l} \Delta=0 \forall_{l=1, \ldots, L-1}$, provided $\mathrm{p}_{T} \neq 0$. Thus a sufficient condition for the maximization of diversity (sum/product) is

$$
\begin{gathered}
\max : \mathrm{d}_{T}^{2}=\|\Phi-\Psi\|_{\mathrm{F}}^{2} \stackrel{!}{>} 0 \\
\max : \mathrm{p}_{T}^{2}=\operatorname{det} \Delta^{\dagger} \Delta \stackrel{!}{>} 0 \\
\forall_{l=1, \ldots, L-1}\left(\Delta^{\dagger} \mu^{l} \Delta=0 \Longleftrightarrow \Delta^{\prime \dagger} \tau^{l} \Delta^{\prime}=0\right)
\end{gathered}
$$

performed over all pairs $\Phi, \Psi$ of code symbols satisfying $\Phi_{\mathrm{mp}}, \Psi_{\mathrm{mp}} \in V_{n_{t} L, K}^{\mathbb{C}}$. So, while the first two conditions are borrowed from the theory of space time codes, the third set of conditions is a serious constraint: The difference vector $\Delta$ of two code symbols $\Phi, \Psi$ has to be perpendicular to its row-wise $l$-th order modulated (resp. its $l$-th order down-shifted) version for $l=1, \ldots, L-1$, reflecting the strong correlation between each $l$-th channel realization in the frequency domain. The 
demanded orthogonality forces the best possible (frequency) diversity induced by the multipath delay spread.

The homogeneity of the diversity (6) permits the interpretation of (9) as a constraint packing problem on the Stiefel manifold $V_{n_{t} L, K}^{\mathbb{C}}$ : Fully diverse space frequency coding becomes a packing problem in $V_{n_{t}, K}^{\mathbb{C}}$ (due to (9a)) subject to the 'space time' constraint (9b) and the 'space frequency' constraint (9c). For space time codes the corresponding interpretation has been pointed out in [9].

\section{SPACE FREQUENCY CODES FROM SPACE TIME CODES}

As has been pointed out in [1], the design of fully diverse space frequency codes splits into two independent parts, reducing the overall complexity of the problem considerably. First construct a space time code $\tilde{\mathcal{C}}^{\prime} \subset V_{n_{t}, T}^{\mathbb{C}}$ (subject to the first two conditions in (9)) for some $T$. Then find some matrix $A^{\prime}$ satisfying

$$
A_{\mathrm{mp}}^{\prime}=\left(A^{\prime}, \tau A^{\prime}, \ldots, \tau^{L-1} A^{\prime}\right) \in V_{T L, K}^{\mathbb{C}}
$$

The final space frequency code fulfilling (9c) is then given as [1]

$$
\mathcal{C}=\operatorname{FFT}\left(A^{\prime} \tilde{\mathcal{C}}^{\prime}\right)
$$

From this construction the rate $R$ of the space frequency code (neglecting the cyclic prefix) and the rate $\tilde{R}$ of the internal space time code are related by

$$
R=\frac{T}{K} \tilde{R}
$$

from which it is obvious that high rate codes require large block lengths $T$. On the other hand to retain the maximal diversity order $L n_{t} n_{r}$ of the space frequency code $T \leq$ $K / L$ is demanded. Therefore the code design aims at code constructions satisfying

$$
T=\frac{K}{L}
$$

and we concentrate on this case in the sequel.

As a further notational convention we introduce the tilde : for quantities corresponding to the 'inner' space time code $\tilde{\mathcal{C}}^{\prime}$. Recall that $\mathcal{C}$ in (11) has full (frequency and spatial) diversity, if $\tilde{\mathcal{C}}^{\prime}$ is a fully diverse space time code and for large block lengths this is a complex code design problem. Therefore the strategy in this work is to concentrate on the simpler first order design criteria (9a) in the next section and develop some adjustments for full diversity later on (section VI), compare the remark in the last paragraph in section III. Due to the factorization (11) the goal investigated in the next section is to find dense packings in the metric space $\left(V_{n_{t}, T}^{\mathbb{C}}, \tilde{\mathrm{d}}_{T}\right)$.

Let us close this section by some analysis of the frequency diversity generating matrix $A^{\prime}$. Random constructions have been proposed in [1], but here we present a deterministic construction which in addition is especially designed to benefit from a low crest-factor: Set

$$
\lambda=\left(\exp \left(\frac{2 \pi \imath k^{2}}{K}\right)_{k=0}^{K-1}\right)
$$

(it is well-known that this sequence belongs to the set of so-called bi-unimodolar sequences, i.e their FFT is as well unimodular). Then the matrix $U(c)=\left(u_{0}, \ldots, u_{K-1}\right):=$ $\left(c, \tau c, \ldots, \tau^{K-1} c\right)$ with $c=\mathrm{FFT}^{\dagger}\left(\frac{1}{\sqrt{K}} \lambda\right)$ is a circulant matrix which is also unitary. Let us define

$$
A^{\prime}:=\left(u_{0}, u_{L}, \ldots, u_{(T-1) L}\right)
$$

then $\tau^{l} A^{\prime}=\left(u_{l}, u_{L+l}, \ldots, u_{(T-1) L+l}\right)$ and $A_{\mathrm{mp}}^{\prime \dagger} A_{\mathrm{mp}}^{\prime}=\mathbf{1}$ as desired. This choice of $\lambda$ distributes the transmit energy equally in the time domain (compare [10] and references therein for an account to the construction of sequences with zero autocorrelation sidelobes and zero crosscorrelation). In general this construction scheme provides a real $K$ dimensional parameter space $\lambda_{k}=e^{\imath \phi_{k}}, 0 \leq \phi_{k} \leq 2 \pi, k=$ $0, \ldots, K-1$ for $A^{\prime}$.

\section{SPACE TIME PACKINGS FROM SPHERICAL CODES}

The Stiefel manifold is canonically a homogeneous space $\left(U(K) \stackrel{\pi}{\longrightarrow} V_{n_{t}, K}^{\mathbb{C}} ; U\left(K-n_{t}\right)\right)$ : The canonical left multiplication of $n_{t}$-frames in $\mathbb{C}^{K}$ by unitary $K \times K$ matrices transforms each pair of $n_{t}$-frames isometrically (with respect to the metric induced from the unitary group) into each other. Thus the action of the unitary group $U(K)$ on $V_{n_{t}, K}^{\mathbb{C}}$ is transitive and establishes the canonical diffeomorphism

$$
V_{n_{t}, K}^{\mathbb{C}} \cong U(K) /\left(\begin{array}{lc}
\mathbf{1} & \mathbf{0} \\
\mathbf{0} & U\left(K-n_{t}\right)
\end{array}\right)
$$

Homogeneity is the natural generalization of spherical symmetry: At any point the manifold 'looks the same' i.e. for any pair $(\Phi, \Psi)$ of points in $V_{n_{t}, T}^{\mathbb{C}}$ there exists an isometry (with respect to the Riemannian metric structure) which takes $\Phi$ into $\Psi$. In contrast to spheres the sectional curvature is no longer constant, but remains non-negative and bounded from above. Since positive curvature contracts distances, the homogeneity $V_{n_{t}, T}^{\mathbb{C}}$ suggests certain (with sectional curvature equal to the upper bound just mentioned) $D$-dimensional spheres as simple comparison spaces,

$$
D:=\operatorname{dim}_{\mathbb{R}} V_{n_{t}, T}^{\mathbb{C}}=n_{t}\left(2 T-n_{t}\right)
$$

Any code on such a sphere can be transformed into a code on the Stiefel manifold without shortening its geodesic distances or volumes (compare [11] for details).

In practice the following has been done. By homogeneity in both spaces one 'base' point has been fixed, e.g. the north pole $N$ of the sphere and the point $\left(\begin{array}{l}\mathbf{1} \\ \mathbf{0}\end{array}\right)$ in the Stiefel manifold. All points on the respective manifold can be equivalently addressed in the corresponding tangent space (with respect to the fixed base point) by utilizing the so-called exponential map, which turns radial tangent lines isometrically into geodesics emanating from the base point. Representing the Stiefel manifold as a $U(T)$-homogeneous space and similarly the sphere as

$$
S^{D} \cong O(D+1) / O(D)
$$

( $O(D)$ denoting the group of $D \times D$ orthogonal matrices), the exponential map exp coincides with the matrix exponential $\exp : A \longmapsto \sum_{k} A^{k} / k$ !. Restricting exp to the ball with radius $\pi$, exp becomes a diffeomorphism onto the corresponding manifolds up to a set of measure zero. Neglecting this sparse set, we have now a representation of the sphere and the Stiefel 
manifold in terms of their tangent space at the chosen base point. Choosing some orthonormal base $\left\{Y_{i}\right\}$, resp. $\left\{X_{i}\right\}$ in each of tangent spaces induces a canonical mapping from (the tangent space of) the sphere to the (tangent space of the) Stiefel manifold by

$$
\sum_{k} v^{k} Y_{k} \longmapsto \sum_{k} v^{k} X_{k}
$$

This mapping is an isometry of tangent spaces and due to curvature non-radial distances will be contracted to an extend which is determined by the sectional curvature upper bound in the Stiefel manifold. Moreover the geodesic distance is only an approximation to the 'chordal' distance $\tilde{\mathrm{d}}_{T}$ of the receiver. But it can be shown, that both distance measures are (locally) equivalent [11]. In summary, spherical codes (for example from MWBE sequences in CDMA systems [4]) can be transformed into space time codes, whereas spherical minimal distances are possibly contracted, but only to an extend, which is determined by the (bounded) curvature of the Stiefel manifold and the local equivalence of the distance measures.

\section{LATTICE SPHERICAL SPACE FREQUENCY CODES AND DIVERSITY ROTATION}

The construction of high rate codes in systems with large block lengths or large number of subcarriers suffers from the increasing (brute force) decoding complexity, which becomes intractable soon. To fight this, additional structures in the code design need to be exploited. One way of embedding (algebraic) structure is provided by the design algorithm for wrapped spherical codes [5]: This algorithm uses a clever and computationally simple one-to-one, distance expanding mapping from $\mathbb{R}^{D}$ to the sphere $S^{D} \subset \mathbb{R}^{D+1}$ to map lattices on (each hemi-)sphere. Having this map and its inverse at hand we can describe the overall encoding and decoding procedure by Algorithm 1. Note that the lattice encoding

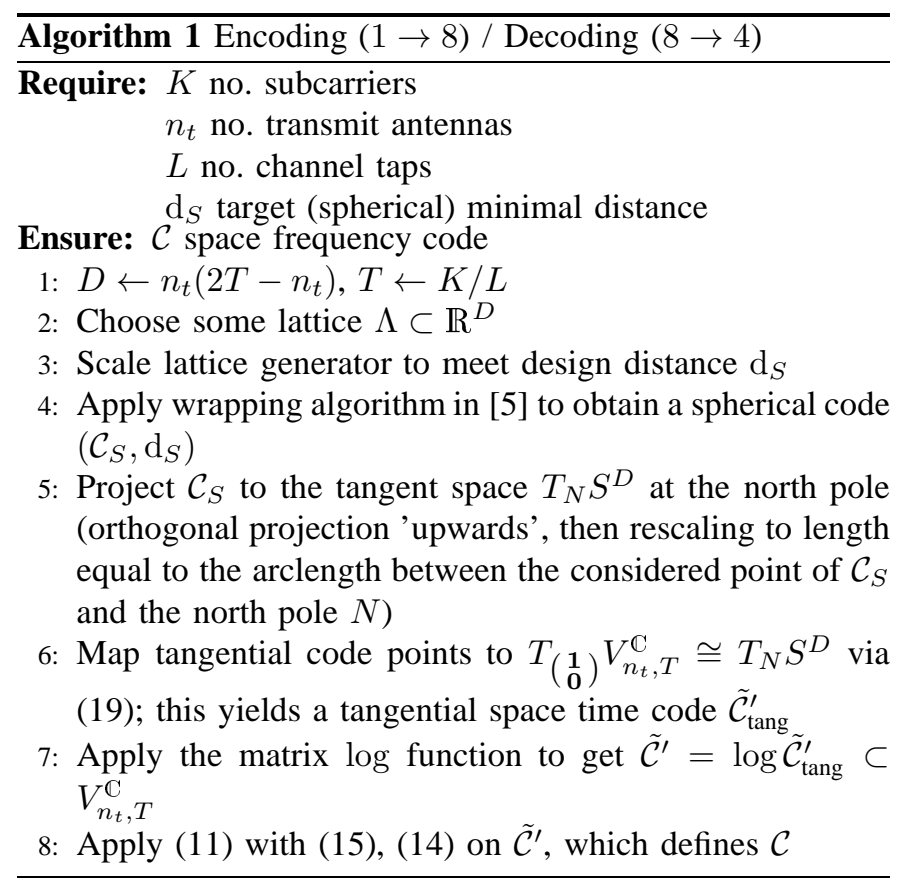

scheme permit large families of code constructions based on (apart from the standard cubic lattices $Z_{n}$ ) the root lattices (and their duals) $A_{n}\left(A_{n}^{*}\right), D_{n}\left(D_{n}^{*}\right)$ in various dimensions $n$, the exceptional lattices $E_{6,7,8}$, the Coxeter-Todd lattice $K_{12}$, the Barnes-Wall lattice $\Lambda_{16}$, and the Leech lattice $\Lambda_{24}$, compare [12] for details. The choice of the design distance $\mathrm{d}_{S}$ trades minimal distance for rate. For decoding just reverse the encoding algorithm until step 4 and apply some lattice decoding afterwards. Note that this procedure is suboptimal in general due to some points possibly getting lost in step 4 of the encoding algorithm (compare [13]). But in the high rate regime it is expected, that this possible performance loss becomes negligible. Furthermore some efforts have been made to compensate the 'buffer regions' ([13]) in part: These regions originally placed as separating annuli on the sphere to guarantee the designed minimal distance $\mathrm{d}_{S}$ have been reinspected whether formerly rejected 'buffered' code points lie at a distance at least $\mathrm{d}_{S}$ from all other code points on the sphere.

A common problem of the proposed lattice based construction is the inherent rigidity of lattices. Although there is some control over the minimal distances, there is no direct control on the diversity order, i.e. the diversity product $\mathrm{p}_{F}$. To overcome the possibility of diversity loss several rotated versions of the lattices have been produced as a preprocessing step of the encoding algorithm. Of course this procedure does not affect the decoding performance. As the axis of rotation the 'diagonal' $e$ defined as the sum of all basis vectors of the respective lattice has been taken. Denote by $W_{e}$ the orthogonal matrix with $e_{1}=W_{e} e$ whereas $e_{1}=(1,0, \ldots, 0)^{t}$. Set $\mathbf{0}=(0, \ldots, 0)^{t} \in \mathbb{R}^{D-1}, R_{1}=\left(\begin{array}{cc}1 & \mathbf{0}^{t} \\ \mathbf{0} & R^{\perp}\end{array}\right)$ and define $R^{\perp}=\exp (\alpha X), \alpha \in \mathbb{R}$, with $X$ being the antisymmetric $(D-1) \times(D-1)$ matrix with ones on its upper triangular part. Then $R_{1}$ is a rotation of $\alpha$ degrees about the axis $e_{1}$ and $R(\alpha)=W_{e}^{t} R_{1} W_{e}$ has $e$ as its axis of rotation.

\section{Simulations AND DiscUSSION}

For the simulations the channel was modeled for $n_{t}=2$ transmit antennas and $L=2$ taps and for simplicity at the receiver maximum likelihood decoding has been performed. We constructed space time codes with block length $T=4$, corresponding to lattice dimension $D=12$, compare (17). In order to obtain full (frequency) diversity for a scenario with $L=2$ channel taps these codes are designed for systems with $K=L T=8$ subcarriers. As a reference, an Alamouti based space time/frequency code of comparable rate has been simulated. Since the block length of the (space time) Alamouti scheme is 2 this code family is well adapted to MIMOOFDM scenarios with $K=2 L=4$ subcarriers. Other choices of $K$ (smaller) would destroy diversity or (larger) add redundancy, which lowers the rate and would not give fair comparisons. Note that the restriction on $K$ for the Alamouti scheme underlines the need for new coding schemes, which are capable of meeting more general system requirements $\left(K, L, n_{t}\right)$. The spherical space frequency codes proposed in this work achieve this by appropriately choosing the spherical dimension $D$. 
Application of Algorithm 1 produced (by the prescription of the spherical minimal distance) two sets of spherical space frequency codes. The first set consists of very high rate codes based on the lattices $K_{12}, D_{12}, K_{12}$ again, and $A_{12}$ with (space frequency) rates $R=1.95,1.71,1.65,1.64$. They will be compared to the 8-PSK modulated Alamouti scheme of (space frequency) rate $R=1.5$. The second set consists of medium rate codes based on the lattices $K_{12}, Z_{12}$, and $A_{12}$ with rates $R=1.32,1.15,1.04$ which are compared to the QPSK Alamouti scheme of (space frequency) rate $R=1$. All these lattice have been rotated as described in the previous section by an angle $\alpha=\pi / 2$ for a better diversity gain. Figure 1 shows the simulation results.

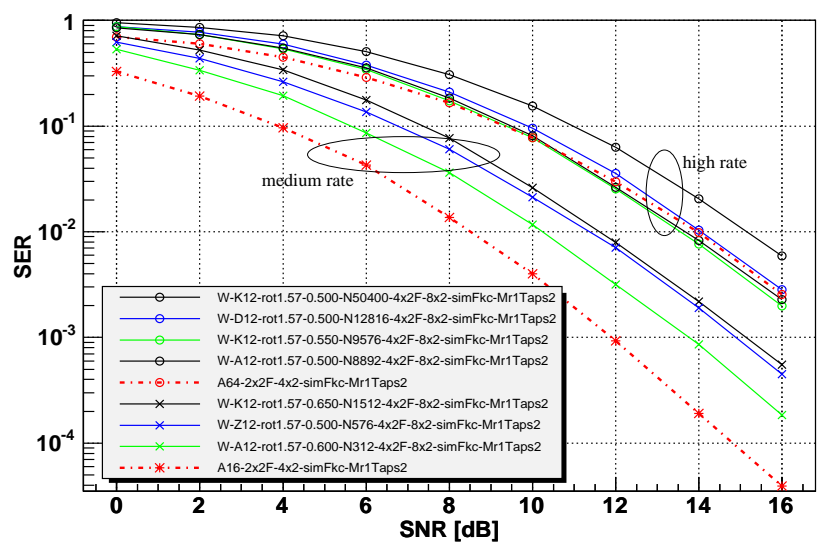

Fig. 1. Symbol error rate for spherical space frequency codes compared to corresponding Alamouti (8-PSK,QPSK) schemes

The simulations demonstrate that in the high rate regime the spherical space frequency codes outperform the corresponding Alamouti scheme. Moreover the Alamouti code has the lowest (space frequency) rate in this comparison.

For medium rates the picture changes. Here the Alamouti scheme (although the code with the lowest rate in the simulation) still outperforms the new spherical space frequency codes. Nevertheless, due to the lattice rotation all codes seem to have full diversity, even the qubic $Z_{12}$ lattice performs not too bad.

\section{CONCLUSIONS}

In this work a class of variable rate, fully diverse spherical space frequency codes based on the scheme [1] has been proposed, which applies to arbitrary channel parameters, i.e. no. of subcarriers $K$, no. of channel taps $L$, no. of transmit antennas $n_{t}$. The possible rates are determined by the choice of the underlying lattice and its designed minimal distance (obtained by an appropriate scaling of the lattice). This makes the constructions rather flexible to meet a wide range of applications. In particular very high rate codes (up to $R=1.95$ for an $8 \times 2$ space frequency code in the examples) can be constructed in this way. The diversity order can be increased by some lattice rotation and in the examples full diversity has been achieved. The multipath diversity controlled by the matrix $A(15)$ has been chosen with respect to an advantageous effect on the power distribution among the subcarriers. The simulations demonstrate satisfactory performance results (at least in the high rate regime), and the lattice based code design offers the possibility of moderate decoding complexity.

However improvements of the code design and its complexity seem to be possible by careful analysis of the encoding algorithm. The connection of space time/frequency codes with spherical codes/WBE sequences in CDMA systems comes as a surprise, made possible by a differential geometric analysis for a (first order) sphere packing approach to space time/frequency coding. This offers a new direction in future research efforts.

\section{REFERENCES}

[1] H. Bölcskei, M. Borgmann, and A. Paulraj, "Space-frequency coded MIMO-OFDM with variable multiplexing-diversity tradeoff," submitted to IEEE Trans. Inform. Theory, 2004.

[2] Z. Liu, Y. Xin, and G. Giannakis, "Space-time-frequency coded OFDM over frequency-selective fading channels," IEEE Trans. Signal Processing, vol. 50, Oct. 2002.

[3] J. Massey and T. Mittelholzer, "Welch's bound and sequence sets for code-division multiple access systems," in Sequences 2, Methods in Communication, Security and Computer Science, U. V. R. Capocelli, A.De Santis, Ed. Springer-Verlag, 1993.

[4] R. W. Heath Jr., T. Strohmer, and A. J. Paulraj, "On quasi-orthogonal signatures for CDMA systems," IEEE Trans. Inform. Theory, 2002, (submitted).

[5] J. Hamkins and K. Zeger, "Asymptotically dense spherical codes — part I: Wrapped spherical codes," IEEE Trans. Inform. Theory, vol. 43, no. 6, 1997.

[6] T. Strohmer and R. Heath, "Grassmannian frames with applications to coding and communication," Appl. Comp. Harmonic Anal., vol. 14, no. 3, pp. 257-275, 2003.

[7] B. Hochwald and T. Marzetta, "Capacity of a mobile multiple-antenna communication link in Rayleigh flat fading," IEEE Trans. Inform. Theory, vol. 45, pp. 139-157, 1999.

[8] — - "Unitary space-time modulation for multiple-antenna communications in Rayleigh flat fading," IEEE Trans. Inform. Theory, vol. 46, pp. 543-565, 2000.

[9] O. Henkel, "Geometrical relations between space time block code designs and complexity reduction," submitted to IEEE Trans. Inform. Theory, 2003. [Online]. Available: $\mathrm{ftp} / / / \mathrm{ftp}$. hhi.de/henkel/decomposition.ps

[10] S. Stanczak, G. Wunder, and H. Boche, "On pilot-based multipath channel estimation for uplink CDMA systems: The overloaded case," IEEE Trans. Signal Processing, 2005, to be published.

[11] O. Henkel, "Sphere packing bounds in the Grassmann and Stiefel manifolds," IEEE Trans. Inform. Theory, (accepted).

[12] J. Conway and N. Sloane, Sphere Packings, Lattices and Groups, 2nd ed. Springer-Verlag, 1992.

[13] J. Hamkins, "Design and analysis of spherical codes," Ph.D. dissertation, University of Illinois at Urbana-Champaign, 1996. 